\title{
Entire crystallization process of Lennard- Jones liquids: A large-scale molecular dynamics study
}

Cite as: J. Chem. Phys. 152, 054903 (2020); https://doi.org/10.1063/1.5139574

Submitted: 21 November 2019 . Accepted: 22 January 2020 . Published Online: 04 February 2020

Wenze Ouyang ${ }^{D}$, Bin Sun, Zhiwei Sun, and Shenghua Xu (D)
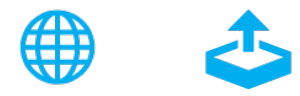

\section{ARTICLES YOU MAY BE INTERESTED IN}

\section{Machine learning for interatomic potential models}

The Journal of Chemical Physics 152, 050902 (2020); https://doi.org/10.1063/1.5126336

Exploring the landscape of Buckingham potentials for silica by machine learning: Soft vs hard interatomic forcefields

The Journal of Chemical Physics 152, 051101 (2020); https://doi.org/10.1063/1.5136041

Dynamic heterogeneity and collective motion in star polymer melts

The Journal of Chemical Physics 152, 054904 (2020); https://doi.org/10.1063/1.5135731

\section{Lock-in Amplifiers up to $600 \mathrm{MHz}$}
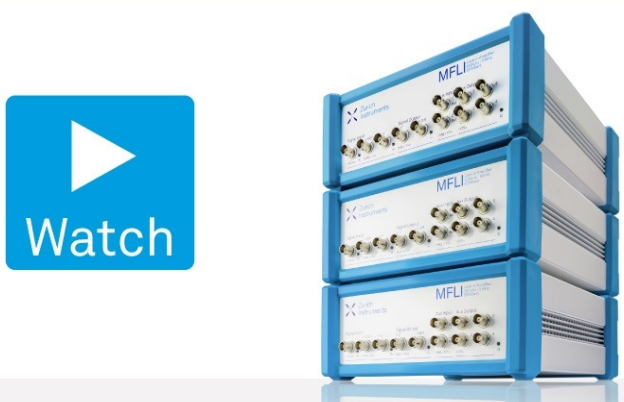


\title{
Entire crystallization process of Lennard-Jones liquids: A large-scale molecular dynamics study
}

Cite as: J. Chem. Phys. 152, 054903 (2020); doi: 10.1063/1.5139574

Submitted: 21 November 2019 • Accepted: 22 January 2020 •

Published Online: 4 February 2020

Wenze Ouyang, ${ }^{1, a)}$ (Din Sun, ${ }^{2}$ Zhiwei Sun, ${ }^{1}$ and Shenghua $X u^{1,3, b)}$ (D)

\begin{abstract}
AFFILIATIONS
${ }^{\top}$ Key Laboratory of Microgravity (National Microgravity Laboratory), Institute of Mechanics, Chinese Academy of Sciences, Beijing 100190, China

${ }^{2}$ School of Materials and Chemical Engineering, Zhongyuan University of Technology, Zhengzhou 450007, China

${ }^{3}$ School of Engineering Science, University of Chinese Academy of Sciences, Beijing 100049, China
\end{abstract}

a) Author to whom correspondence should be addressed: oywz@imech.ac.cn

b) Electronic mail: xush@imech.ac.ch

\begin{abstract}
By using a graphics processing unit-accelerated parallel algorithm on a compute unified device architecture platform, we perform large-scale molecular dynamics simulations in a Lennard-Jones system to observe the entire crystallization process, including metastable stage, critical nuclei formation, and the stage of crystal growth. Although the intermediated precursors that play a role in determining the polymorphs are predominantly bcc ordered, the polymorph selection is rather different at different stages. The precursors that have a relatively high orientational order will be on average in a denser region than uniform liquids, but microscopically the crystal nucleation happens without a density change. The average density of nuclei first increases significantly, and then almost keeps independent on the crystallite size after the growing post-critical nucleus becomes large enough. With such a large enough system, the crystal growth rate is able to be calculated directly by doing a linear fit to the temporal evolution of growing crystallite size. The obtained value of the growth rate indicates that the actual crystal growth in the Lennard-Jones system where the crystal-liquid interface has several kinds of structures is possibly driven by both collision-controlled and diffusion-controlled mechanisms.
\end{abstract}

Published under license by AIP Publishing. https://doi.org/10.1063/1.5139574

\section{INTRODUCTION}

Crystallization is a typical process where the supercooled liquid passes through some scenarios to transform into a crystal eventually. Due to its practical consequences in science, technology, and our daily life, it has attracted a lot of interests in scientific research. ${ }^{1}$ The crystallization process, which is physically a first-order phase transition, usually has three stages: (I) the metastable stage where many subcritical nuclei form and dissolve, (II) the stage of critical nuclei formation, and (III) the stage of crystal growth. So far, experimental, theoretical, and numerical studies on the crystallization have been done extensively, but the understanding of the crystallization process is far from being complete as there are still a lot of debates over its subtle details and the underlying mechanism.

Among the countless studies of the crystallization process, computer simulation is a good way because it can provide a detailed picture of the nuclei formation and subsequent crystal growth on a microscopic level. ${ }^{4}$ When considering the methods of computer simulation, a natural choice is the so-called brute-force molecular dynamics (MD), in which one would just cool the system to below the melting temperature and then simply wait for the crystal nucleation to happen. Until now, the brute-force MD has been a popular and robust method to study the crystallization process as it is straightforward and can directly provide a "real" temporal evolution. Although some enhanced sampling methods where specific and complex computational techniques that artificially accelerate the emergence of the critical nucleus are proposed to observe the nucleation event within a much shorter time scale, ${ }^{2}$ they in principle alter the dynamics of a system.

As for the simulation of the crystallization process, stage (I) and stage (II) have been well studied, but not stage (III) because it requires a large enough system whose computational cost is very expensive. Actually, this is also related to a long-standing disadvantage that has to be considered in simulation, i.e., the finite size effects 
due to the small number of particles or molecules. To eliminate the artifacts from finite size effects, people have not yet agreed on exactly how many particles should be used in a typical MD simulation of crystallization. In 1986, Honeycutt and Andersen reported that the critical nucleus size and nucleation rate were largely dependent on the size of the simulated Lennard-Jones (LJ) system, i.e., the critical nucleus size and nucleation rate were smaller in a small system than those in a large system, indicating that artifacts in the crystallization process might be produced by the small system size and periodic boundary conditions. ${ }^{5}$ A few years later, Swope and Andersen continued to study the crystal nucleation of the LJ system with a sufficiently large number of particles and their results suggested that more than $10^{4}$ particles would avoid finite-size effects. ${ }^{6}$ The work of Huitema ${ }^{7}$ seemed to support the outcome of Swope and Andersen as they studied the nucleation rate of the LJ system. Yet, when concerning the growth of post-critical nuclei, one should take into account additional artifacts produced by the finite system size. As is known, a solid crystalline region that emerges and grows in a liquid often has a structure with crystal particles and an attached liquid-like interface and extends to a distance. Especially, when the solid crystalline reaches a rather large size where its extending distance exceeds half the simulation cell length, the nucleus will definitely "feel" the image of itself under the periodic boundary conditions. This may influence some observations such as the rate of crystal growth and the subsequent structure formation related to the polymorph selection. In 2006, Streitz et al. observed the solidification in molten tantalum and showed that the independence from finite-size effects during the entire nucleation and growth process should need more than $10^{6}$ atoms. ${ }^{8}$ In other words, in order to circumvent the artifacts from finite-size effects and produce an accurate model of reality, the system size used for the simulation of the entire crystallization process had better be much larger than normal expectation.

In this work, we will perform large-scale brute-force MD simulations via a graphics processing unit (GPU)-accelerated parallel algorithm implemented on a Compute Unified Device Architecture (CUDA) platform, where more than $10^{6}$ particles are used to observe the entire crystallization process. As an illustration, the model LJ system is considered here based on the following reasons. First, the LJ potential has a simple functional form which facilitates evaluations. Second, as the LJ system plays an important role in statistical physics and has become one of the most widely studied model systems, ${ }^{9}$ much data, especially its accurately characterized liquid-solid phase diagram, are already available, ${ }^{10-12}$ which makes the simulation of solidification explicit. Third, there are already a lot of previous numerical studies on the crystal nucleation and its underlying nucleation mechanism, where a small number of particles are mostly used so that we can compare our results of the large system size with previous observations conveniently as well as discover something new relevant to crystallization. In most previous simulations of LJ crystallization, the stage of crystal growth is not well studied somewhat due to small system size, which makes the understanding of the entire crystallization process incomplete. Here, more than $10^{6}$ particles are used in MD simulations, where the system size would be large enough to let the post-critical nucleus in a simulation box extend to fairly long distance before it can "feel" the image of itself. We expect some useful information, especially the observations of crystal growth, can be achieved so as to shed a new insight into the physics of the crystallization process.

\section{DETAILS OF SIMULATION}

As is known, the LJ potential is written as

$$
U\left(r_{i j}\right)=4 \epsilon\left[\left(\sigma / r_{i j}\right)^{12}-\left(\sigma / r_{i j}\right)^{6}\right],
$$

where $r_{i j}$ is the pair distance between the centers of $i$ th particle and $j$ th particle. The parameters $\epsilon$ and $\sigma$ define the strength and length scale of the interaction, respectively. As is common for the simulations of the LJ system, we use the reduced units, where energy unit $\epsilon$, length unit $\sigma$, and the mass of particle $m$ are chosen as the basic units. The cutoff distance of LJ interaction is $r_{c u t}=3.0$ and a long-range correction is added for the calculation of thermodynamic quantities. ${ }^{4,13}$

In order to make the large-scale MD simulations run fast and efficiently, we implement a GPU-accelerated parallel algorithm ${ }^{14}$ via the program of CUDA. ${ }^{15}$ The ensemble of each MD simulation is the NPT ensemble, where the total number of particles $N$, the pressure $P$, and the temperature $T$ are kept constant. We apply the period boundary conditions and integrate the equation of motion by using the velocity Verlet algorithm ${ }^{4,13}$ with the time step $\delta t=0.005$. To control the constant temperature and pressure, we adopt the Berendsen thermostat and barostat. ${ }^{16}$

During the numerical study of crystallization, we need to identify different structures, especially the crystal or solid particles in the simulation box. Here, we distinguish crystal particles from liquid particles using the bond-orientational order parameters. Those bond-orientational order parameters are originally introduced by Steinhardt et al. ${ }^{17}$ and afterward applied by ten Wolde and coworkers $^{18,19}$ for crystal identification. First of all, we should consider a set of nearest neighbors of a particle $i$ we are interested in as all particles $j$. Formerly, the nearest neighbors of a concerned particle are often determined based on a cutoff radius or the assignment of a fixed number of nearest neighbors. Such a straightforward way is actually not appropriate to identify neighborhood arrangement for a lot of systems, especially when concerning long-range interactions. ${ }^{20,21}$ Hence, we adopt the Voronoi tessellation instead of a straightforward method to determine the nearest neighbors, in which the arbitrary parameters are definitely not used. We also normalize bond-orientational order parameters via the surface area of each Voronoi cell, as suggested by Mickel et al. ${ }^{20}$ Assuming that the surface area of the Voronoi cell facet separating particle $i$ and its nearest-neighbor particle $j$ is $A_{i j}$ and the total surface area $A_{t o t}(i)=\sum_{j=1}^{N_{n b}(i)} A_{i j}$, we can define a complex vector $q_{l m}(i)$ for a particle $i$,

$$
q_{l m}(i)=\frac{1}{N_{n b}(i)} \sum_{j=1}^{N_{n b}(i)} \frac{A_{i j}}{A_{t o t}(i)} Y_{l m}\left(\mathbf{r}_{i j}\right) .
$$

$N_{n b}(i)$ is the number of the nearest neighbors of particle $i$ and $\mathbf{r}_{i j}$ is the vector from particle $i$ to $j$. The functions $Y_{l m}\left(\mathbf{r}_{i j}\right)$ are the spherical harmonics, $l$ is a free integer parameter, and $m$ is an integer that runs from $m=-l$ to $m=l$. In order for the identification of solid particles, we take the integer parameter $l=6$ and normalize the complex vector $q_{6 m}(i)$ as

$$
d_{6 m}(i)=\frac{q_{6 m}(i)}{\left[\sum_{m=-6}^{6}\left|q_{6 m}(i)\right|^{2}\right]^{1 / 2}} .
$$


Then, we calculate a scalar product which measures the correlation between neighboring particles $i$ and $j$,

$$
S_{i j}=\sum_{m=-6}^{6} d_{6 m}(i) \cdot d_{6 m}^{*}(j),
$$

where the superscript $*$ indicates complex conjugation. We consider two neighboring particles $i$ and $j$ to be connected if $S_{i j}$ exceeds a given value, typically $S_{i j}>0.7$. When a particle $i$ has at least seven connected neighbors, we can say that it is a solid or crystal particle.

We use the coarse-grained bond-orientational order parameters introduced by Lechner and Dellago ${ }^{22}$ to further determine the structure of each solid particle. Different from Eq. (2), the coarsegrained complex vector $\bar{q}_{l m}(i)$ of particle $i$ is calculated by averaging $q_{l m}$ over all its nearest neighbors as

$$
\bar{q}_{l m}(i)=\frac{1}{N_{n b}(i)} \sum_{k=0}^{N_{n b}(i)} q_{l m}(k)
$$

where $k=0$ means particle $i$ itself. Then we calculate the local order parameters of averaged version

$$
Q_{l}(i)=\left(\frac{4 \pi}{2 l+1} \sum_{m=-l}^{l}\left|\bar{q}_{l m}(i)\right|^{2}\right)^{1 / 2}
$$

and

$$
W_{l}(i)=\sum_{m_{1}+m_{2}+m_{3}=0}\left(\begin{array}{ccc}
l & l & l \\
m_{1} & m_{2} & m_{3}
\end{array}\right) \frac{\bar{q}_{l m_{1}}(i) \bar{q}_{l m_{2}}(i) \bar{q}_{l m_{3}}(i)}{\left(\sum_{m=-l}^{l}\left|\bar{q}_{l m}(i)\right|^{2}\right)^{3 / 2}},
$$

where the term in parentheses is the Wigner $3-j$ symbol. The integers $m_{1}, m_{2}$, and $m_{3}$ run from $-l$ to $l$ with the precondition that $m_{1}+m_{2}+m_{3}=0$. After a crystal particle is identified, ${ }^{18,19}$ we take advantage of the sign of $W_{6}$ and $W_{4}$ to distinguish its symmetry type. ${ }^{23}$ The following criterion is used: the symmetry of a crystal particle is (i) face-centered-cubic (fcc) as $W_{6}(i)<0$ and $W_{4}(i) \leq 0$; (ii) hexagonal close-packed (hcp) as $W_{6}(i)<0$ and $W_{4}(i)>0$; and (iii) body-centered-cubic (bcc) as $W_{6}(i) \geq 0$.

\section{RESULTS AND DISCUSSION}

The phase diagrams, especially the liquid-solid phase diagram of the LJ system have been extensively studied and accurately constructed. It is well known that the thermodynamically stable solid phase of the LJ system has a fcc structure. Besides stable fcc, there are also two kinds of metastable crystal structures, i.e., hcp and bcc. For a given melting temperature $T_{m}$, here we use the fitting formula of the coexisting curve obtained by van der $\operatorname{Hoef}^{11}$ to calculate the coexistence pressure

$$
P_{\text {coex }}=\beta^{-5 / 4} \exp \left(-0.4759 \beta^{1 / 2}\right)\left[16.89-7.2866 \beta-2.9895 \beta^{2}\right],
$$

where $\beta=1 / k_{B} T_{m}$. When performing a brute-force MD simulation, we should bear in mind that the temperature must be below the melting temperature to ensure a large enough supercooling. We use a very large number of particles $N=4 \times 64^{3}=1048576$, and find that the LJ liquids can crystallize under a fairly lower supercooling than those with small number of particles. Here, we take the supercooling as $28 \%$, in order that the coalescence of nuclei seldom happens and the post-critical nuclei can grow to a rather large size before exceeding the boundary of the simulation box. The melting temperature we take is $T_{m}=2.0$ (the temperature of simulation is $T=1.44$ for a supercooling of 28\%) and the pressure $P=21.23$ can be calculated immediately by Eq. (8). For a better statistics, we have performed 100 sets of MD simulations independently. Among those 100 generated trajectories, we have seen that 74 trajectories succeed in crystallization. In the following, we will describe in detail the observations of the entire crystallization process.

\section{A. Precursor-mediated crystallization}

The degree of crystallinity $f_{c}$ can be defined as the number of solid particles divided by the total number of particles $N$. In the top of Fig. 1, we show the temporal evolution of $f_{c}$ during a typical
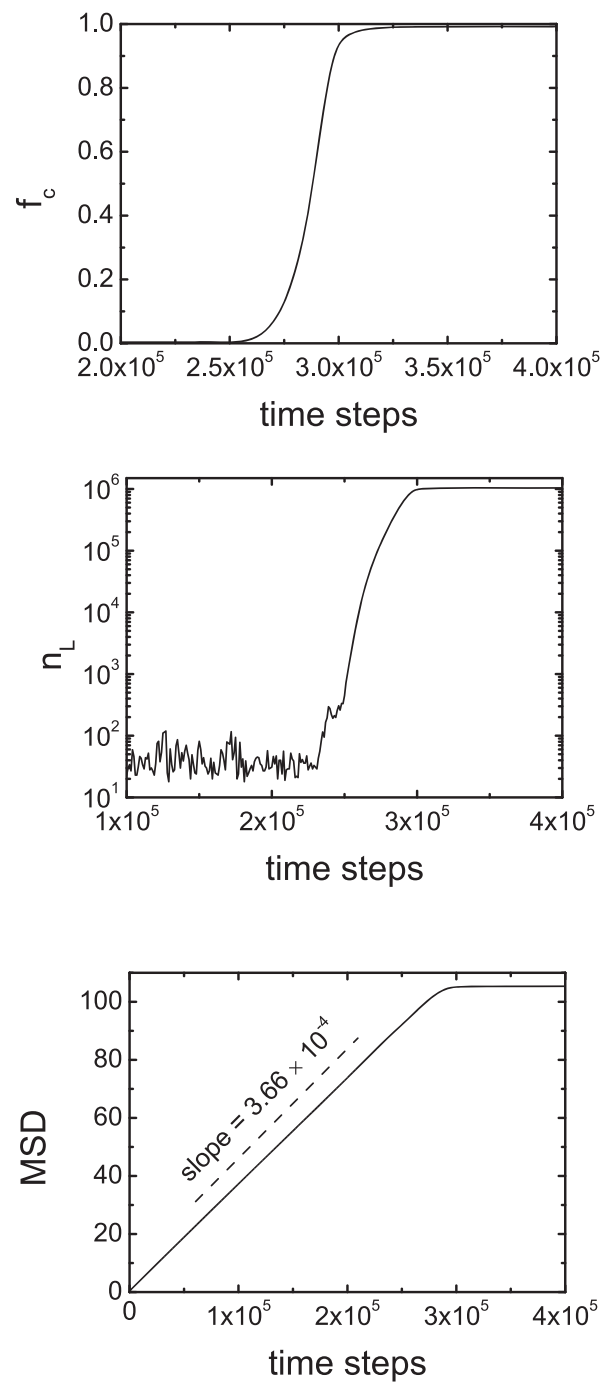

FIG. 1. Crystallization process in a typical MD simulation for $T=1.44$ (the melting temperature $T_{m}=2.0$ and the supercooling is $28 \%$ ) and $P=21.23$. Top: Degree of crystallinity (crystal fraction) $f_{c}$. Middle: Size of the largest crystal nucleus $n_{L}$. Bottom: Mean square displacement (MSD). 
crystallization process. Obviously, the system at such a relatively small supercooling we chose has been a steady-state metastable fluid for a rather long time (more than $2 \times 10^{5} \delta t$ ). In the stage of metastable fluid, the fraction of solid particles is very small and most of the particles that are in liquid environments can move freely (see the evolution of mean square distance in the bottom of Fig. 1). After the system succeeds in the formation of a critical nucleus, more and more liquid particles transform into solid particles so that $f_{c}$ increases significantly. Eventually, the system can almost completely crystallize as $f_{c}$ nearly reaches 1.0 .

To directly see the crystallization process, we plot some snapshots of the typical MD trajectory (corresponding to Fig. 1) via the software Ovito. ${ }^{24}$ As there are so many (more than one million) particles, we only display the crystal particles for a clear view. The crystal particles with different structures are depicted as different colored spheres. As is shown in Fig. 2(a), many small nuclei form and dissolve in the metastable fluid. These subcritical nuclei are indeed not spherical as assumed in the classic nucleation theory (CNT), but seem to be diffusive. Only when one of these nuclei reach a critical size [see Fig. 2(b)], can it grow up until the whole system crystallize. During this typical crystallization process, we clearly see that the

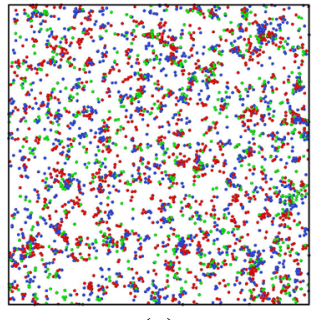

(a)

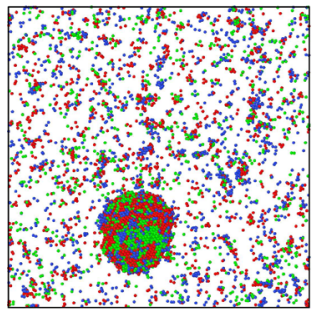

(c)

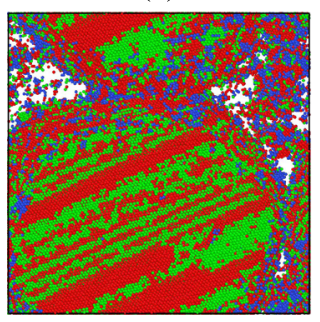

(e)

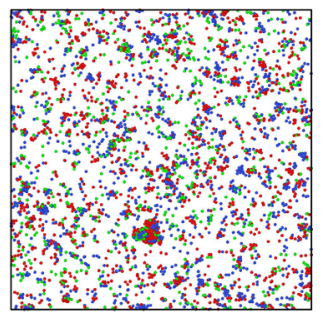

(b)

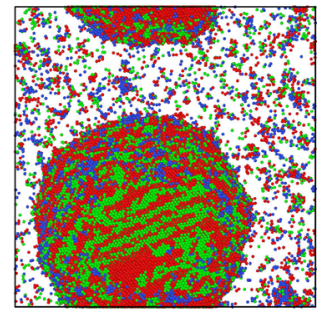

(d)

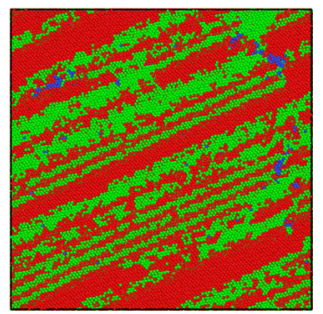

(f)
FIG. 2. Snapshots of a typical crystallization process, corresponding to Fig. 1. Red, green, and blue spheres represent fcc, hcp, and bcc crystal particles, respectively. Liquid particles are not displayed for clear view. (a) $t=2 \times 10^{5} \delta t$. (b) $t=2.4 \times 10^{5} \delta t$. (c) $t=2.6 \times 10^{5} \delta t$. (d) $t=2.8 \times 10^{5} \delta t$. (e) $t=2.9 \times 10^{5} \delta t$. (f) $t=4.0 \times 10^{5} \delta t$. crystallization starts from one critical nucleus but not many. As the post-critical nucleus grows larger and larger, its size may increase until it can even feel the image of itself or even touch itself [see Figs. 2(d) and 2(e)]. When concentrating on the structure of the growing largest nucleus, we investigate that its surface has most of the bcc crystals, while the inside structures are predominantly fcc and hcp crystals. This observation is in agreement with the previous results of ten Wolde and co-workers. ${ }^{18,19}$ In Subsection III B, we will analyze more deeply the polymorphs in the nuclei, especially in the growing post-critical nuclei. It can be seen from Fig. 2(f) that there are mostly fcc and hcp crystals and very few bcc crystals left at the end of crystallization.

It has already been found that a coarse-grained bondorientational order parameter $Q_{6}$ [see Eq. (6)] plays an essential role in the crystallization of many kinds of liquids as $Q_{6}$ drives the whole crystallization process. ${ }^{3,25}$ Usually, the crystal nucleation happens in the region of preordered liquids with relatively high $Q_{6}$. Those preordered liquid particles that act as the seeds of crystal nucleation are often defined as precursors. Here, we just simply identify the precursor particles using a threshold value of $Q_{6}$ (typically liquid particles with $Q_{6} \geq 0.25$ ) as previously done. ${ }^{26-28}$ To study the structure of precursors, we use the method similar to the identification of crystal structures, i.e., different precursor structures are identified by their symmetries on the $W_{6}-W_{4}$ plane. For a precursor particle, it is (i) fcc-like as $W_{6}<0$ and $W_{4} \leq 0$; (ii) hcp-like as $W_{6}<0$ and $W_{4}>0$; (iii) bcc-like as $W_{6} \geq 0$. After counting the number and evaluating the fraction of different precursors (Fig. 3), we can see that the precursors during the whole crystallization process have
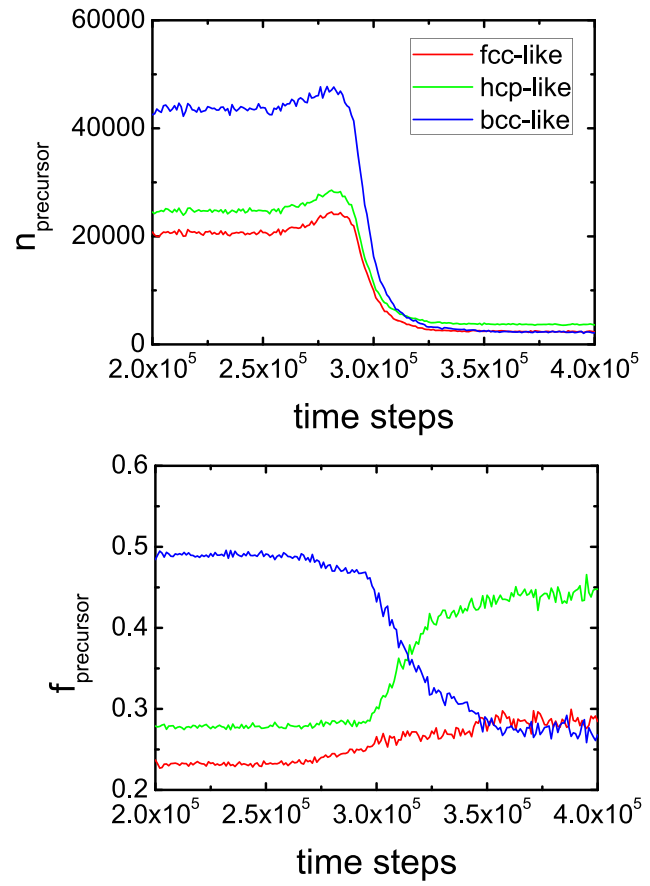

FIG. 3. Evolution of precursors during a typical crystallization process, corresponding to Fig. 1. Top: Number of fcc-like, hcp-like, and bcc-like precursors. Bottom: Fraction of fcc-like, hcp-like, and bcc-like precursors. 
a structure with dominant bcc symmetries, which is also found in other systems. ${ }^{21,23,26,28}$ For a further understanding of the precursormediated scenario, we evaluate the average connected neighbors $\bar{\xi}$ ( $\xi \geq 7$ for solid particles) of precursors. Seen from the top of Fig. 4, the $\bar{\xi}$ of precursors is almost constant at the metastable stage but increases during crystallization due to the increase in the attached precursors on the surfaces of growing crystal nuclei (see the bottom of Fig. 4). Eventually, most of the particles have become crystals and only few precursors that have relatively high $\xi$ are left (see also Fig. 3).

Based on the picture of precursor-mediated crystallization, we describe the entire crystallization process as follows: Starting from an initial random configuration, the system soon become a metastable fluid where many small precursor clusters appear to spread all over the simulation box. Subsequently, some nuclei emerge inside the precursor clusters. However, these nuclei are not stable at all and always dissolve, while some other nuclei form in other places. After waiting for a long enough time, one nucleus fortunately grows up to a critical size and can continue to grow. During the crystal growth, there are also many precursor particles attached on the surface of the growing post-critical nucleus. These precursors, which are not only the seed of crystal nucleation but also the mediated transformation for crystal growth, have the structure of predominantly bcc ordered. Eventually, the growing post-critical nucleus exceeds the boundary of the simulation box and no more transformed precursors attach; so there happens a self-adjusting of the system in which most bcc crystals transform into fcc or hcp crystals.
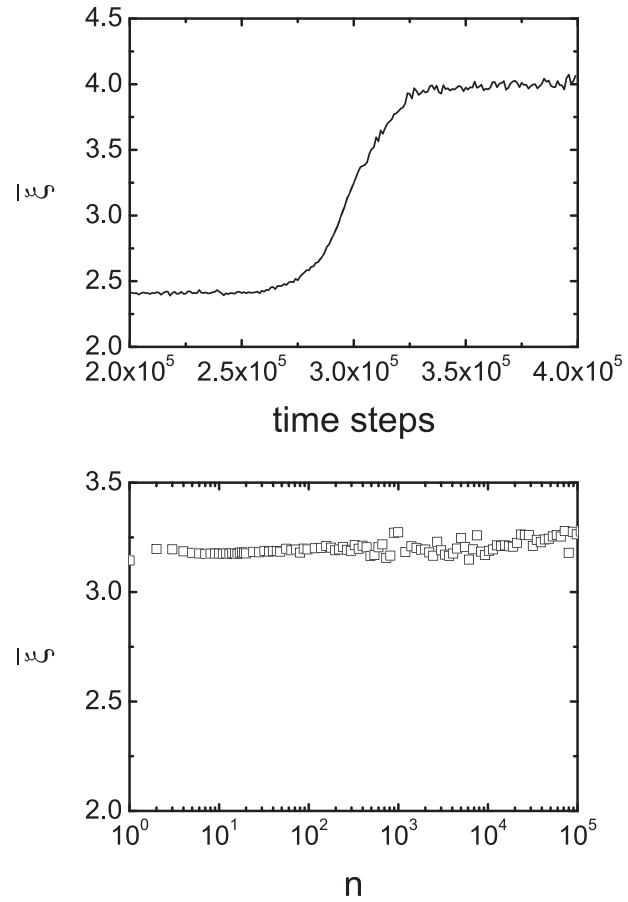

FIG. 4. Average connected neighbors of precursors $\bar{\xi}$. Top: Temporal $\bar{\xi}$ during a typical crystallization process, corresponding to Fig. 1 . Bottom: $\bar{\xi}$ of precursors attached on the surface of nuclei vs the crystallite size $n$.

\section{B. Polymorph selection}

To study the polymorphs of the LJ system during the crystallization process, we apply the method of crystal identification ${ }^{18,19}$ to all of MD trajectories and make a statistic analysis on the composition of each solid cluster. Figure 5 displays the average number of particles and fraction for fcc, hcp, and bcc polymorphs as a function of crystallite size $n$. As expected, the fcc phase is always dominant over hcp and bcc phases during the entire crystallization process. Such a dominant fcc phase, especially during crystal nucleation, has been observed frequently in previous studies. ${ }^{6,18,19,29-31}$ For small nuclei, the number or the fraction of bcc particles seems to be fairly significant (even be close to that of fcc particles for extremely small nuclei). With the increase in the nucleus size, the number of bcc particles begins to increase slower than that of fcc and hcp particles so that the fraction of bcc decreases until it reaches a rather small value. As for the hcp, its fraction decreases slightly first and then increases apparently with the growth of nuclei.

From the observations of Fig. 5, we can say that the polymorph selection is very different at different stages of the crystallization process. At the stage of metastable fluid, the systems bear a lot of small nuclei with different sizes [see Fig. 2(a)] that may dissolve again. Notice here that the crystal nucleus size $n$ in metastable fluid can reach a value more than 100 (see the top of Fig. 6). Concerning the composition of total crystal particles at the metastable stage for a typical MD simulation, fcc particles account for about $40 \%$ and bcc particles account for about $37 \%$, while hcp particles account for
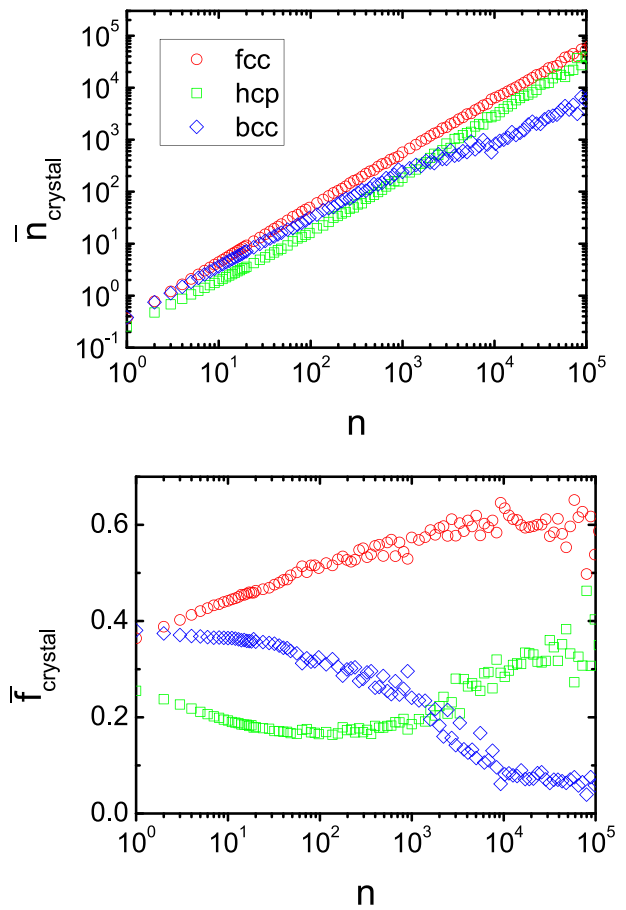

FIG. 5. Average number (top) and fraction (bottom) of different solid particles in the crystal as a function of the total crystal size $n$. Red circles, green squares, and blue diamonds represent fcc, hcp, and bcc polymorphs, respectively. 

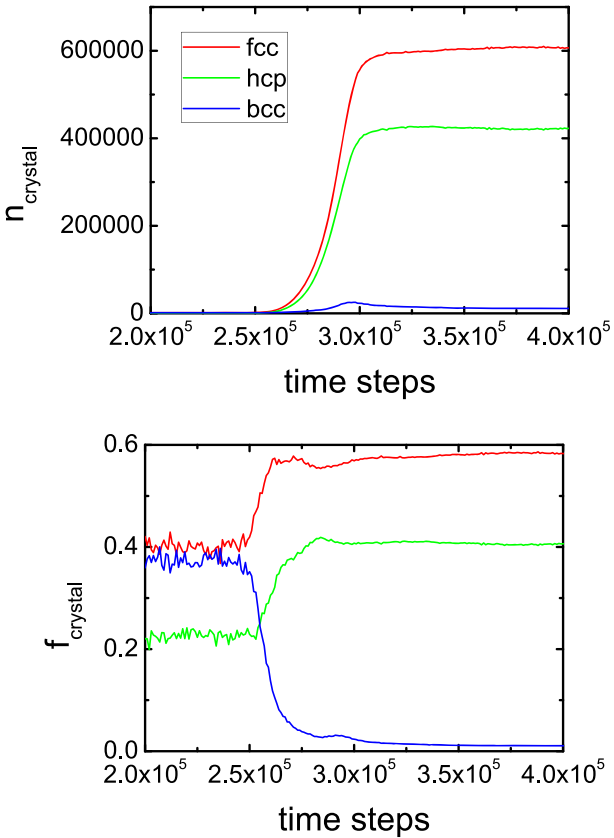

FIG. 6. Evolution of each polymorph during a typical crystallization process, corresponding to Fig. 1. Top: Number of each kind of crystal particles. Bottom: Fraction of each kind of the crystal structure.

about $23 \%$ indicating that the hcp phase plays a minor role in small nuclei (see the bottom of Fig. 6). When the crystallization enters stage (II), one of the nuclei reaches a critical size and can grow subsequently. This nucleus, which is considered to be a critical one, probably has a predominant fcc core with some attached bcc particles and few hcp particles. During the growth of a post-critical nucleus, more and more both fcc and hcp particles are formed at the expense of bcc. Especially, when the size of the post-critical nucleus grows to be very big, the fraction of hcp can eventually reach a significantly large value (close to 0.4 ), while bcc seems to become minor.

For a deeper view of the polymorph selection of the LJ system, it is necessary to answer the question why the composition of crystal nuclei changes at different stages of crystallization. Indeed, this issue is related to the underlying mechanism of crystallization. As already stated, the system holds for a precursor-mediated crystallization process, thus we first need to investigate the precursors attached on the surfaces of crystal nuclei that may probably transform into crystals. In Fig. 7, we present the fraction of different kinds of precursors attached on the surfaces of crystal nuclei as a function of the corresponding nucleus size. During the entire crystallization process, the bcc-like precursors always dominate over the fcc-like and hcp-like precursors, which is in accord with the observations of Fig. 3. For a fairly wide range of nucleus size (up to $n=1000$ that is definitely larger than the critical size), the composition of attached precursor hardly changes. This means that the structure of precursors does not vary much both in metastable fluid and during the nucleation process. After the nucleus grows to a large enough size so that the polymorph varies much, the fraction of the
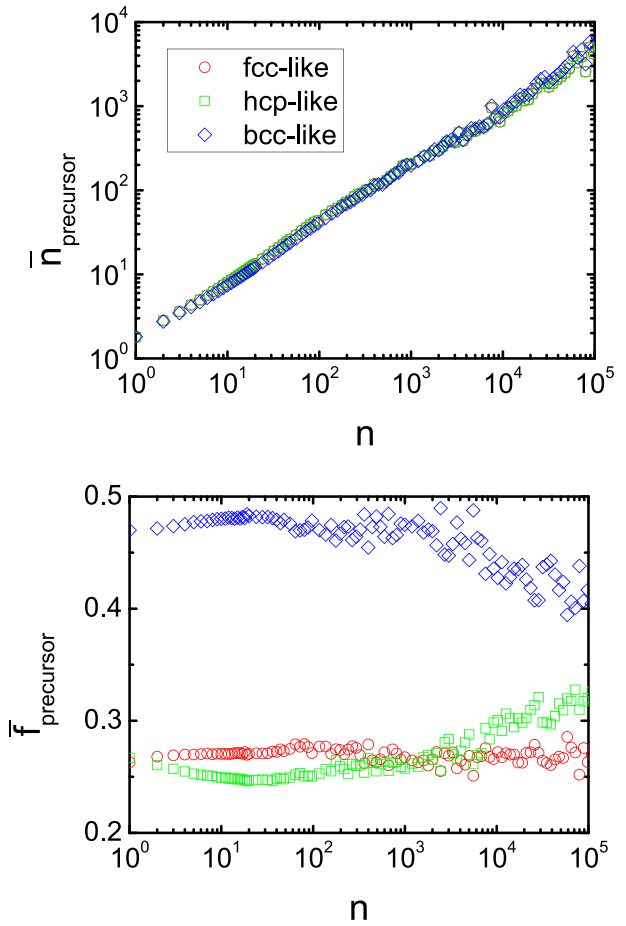

FIG. 7. Average number (top) and fraction (bottom) of different precursor particles attached on the surface of nuclei as a function of the total crystal size $n$. Red circles, green squares, and blue diamonds represent the curves of fcc-like, hcplike, and bcc-like precursors, respectively.

hcp phase increases and the fraction of the bcc phase decreases (see Fig. 5). In return, the crystal composition, especially the one near the nucleus surface influences the newly transformed precursors so as to make the hcp-like and bcc-like precursors vary consistently with the tendency of hcp and fcc crystals.

Knowing the dominant bcc-like structure of the intermediated precursors, we can immediately explain the significant bcc ordering in small nuclei, as it is easier for a bcc-like precursor particle to transform into a bcc crystal particle due to the compatible symmetry. When concentrating on the largest nucleus in crystallizing fluid, we have seen that the bcc crystal particles mostly scatter on the surface of the growing nucleus, especially for a fairly large post-critical nucleus [see, e.g., Fig. 2(c)]. Such a phenomenon was also observed by ten Wolde and co-workers, ${ }^{18,19}$ which has been regarded as the manifestation of the Ostwald's step rule. ${ }^{32}$ During the growth of the crystallite, the bcc phase near its surface can transform into a fcc or hcp phase, while the outside precursors spontaneously join in and become a new bcc phase. As the bcc crystals are more likely to stay on the nucleus surface, it is no wonder that the number of bcc crystal particles increases more slowly than that of fcc and hcp for large nuclei. At the later stage of crystal growth, besides the decrease in the fractional bcc phase, the fraction of the hcp phase becomes significant. This relatively high fraction of the hcp phase is considered to result from the cross nucleation of hcp clusters on the (111) fcc planes. $^{29,30}$ 


\section{Role of density}

There are two order parameters that characterize the liquidsolid phase transition (orientational order and translational order). The orientational order (typically the bond-orientational order parameter $Q_{6}$ ) has been convinced by many previous works to play a key role in the crystallization. The translational order, which can be obtained by computing two-body correlation functions, is in practice found to correlate well with the local density. Thus the local density is often used as a measure of translational order. Up to now, it is still unclear whether the local density plays the same role as the orientational order during the crystallization. For instance, there have been some debates about "density-first" or "bond-orderfirst" in the literature. ${ }^{3}$ For the crystallization of hard spheres, a very recent study suggested a simultaneous increase of density and the bond-orientational order in the early crystal nucleation process. ${ }^{3}$ In the following crystal growth, the crystal density of hard spheres increases with crystallite size. ${ }^{34}$ However, the case is very different in crystallization of soft particles as some studies have indicated that the role of density seems to be trivial. ${ }^{23,27,35}$

Here, we calculate the local density of each particle via Voronoi diagrams, i.e., a particle's density $\rho_{i}=1 / v_{i}$, where $v_{i}$ is the volume of polyhedron subdivided for the particle. Then it is likely to address the interplay between the density and orientational order. In Fig. 8, we show the correlation between the local density and orientational order at the stage of metastable fluid. As is shown in the top of Fig. 8, we average over all the local densities of particles whose
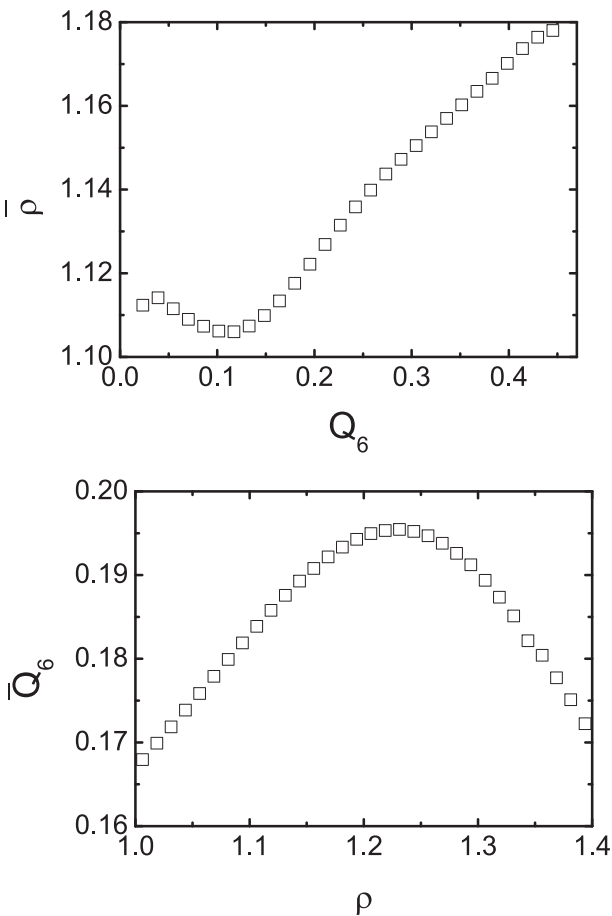

FIG. 8. Relation between local density and coarse-grained bond-orientational order parameter $Q_{6}$ in metastable fluid. Top: average density as a function of $Q_{6}$. Bottom: average $Q_{6}$ as a function of local density. orientational order has the desired value. Apparently, there is a weakly linear coupling relation between the average density $\bar{\rho}$ and $Q_{6}$ for a relatively high order, which is already observed in the crystallization of hard spheres. ${ }^{34}$ This linear term indicates that the regions of high orientational order will on average have higher density than the uniform fluid. However, the opposite is not sure to be true, i.e., the regions of high density are not necessary on average to have a high orientational order. In the bottom of Fig. 8, we present the average orientational order $\bar{Q}_{6}$ for a set of local density values. Indeed, higher density does not correspond to higher orientational order because the average orientational order is a non-monotonic function of the local density. This implies that there would be some structures that have high local density but do not have a high bondorientational order. The icosahedral clusters, as an inhibitor to crystallization, ${ }^{36}$ are considered to belong to such a kind of structure, as they correspond to very high local packing of particles, but are incompatible with the crystalline bond-orientational order.

We also investigate the temporal evolution of average densities for different structures (uniform liquids with $Q_{6}<0.25$, precursors, and crystals) during a typical crystallization process. Seen from Fig. 9, both precursors and uniform liquids have on average a nearly constant density during the entire crystallization process. The average density of precursors is a little higher $(\sim 2.4 \%)$ than that of uniform liquids. Notice that the unnatural behavior in the tail of curves is due to the fact that most liquid particles have transformed into crystals and the system gets into a rearrangement. Obviously, the variation of crystal density is different from that of liquid density. At the stage of metastable fluid, where there are a lot of small nuclei, the average density of crystals is very close to that of precursors. As the precursor acts as the intermediate structure of crystallization, we can conclude that when the liquid-solid transition happens there is almost no density change microscopically. Such a conclusion was also found to hold true for other systems such as hard spheres and soft particles. ${ }^{23,26,34}$ At the stage of crystal nucleation and growth, the average density of crystals increases remarkably until its value almost reaches a plateau.

In order to have a further understanding of the role of density during crystal nucleation and growth, we calculate the average density of crystallites with different cluster sizes, as is displayed in Fig. 10. The average density of crystallites evidently increases with

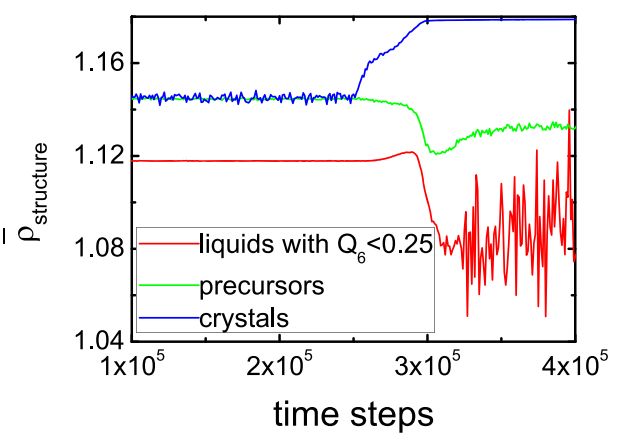

FIG. 9. Temporal evolution of average density for different structural particles (uniform liquids, precursors, and crystals) during a typical crystallization process, corresponding to Fig. 1. 


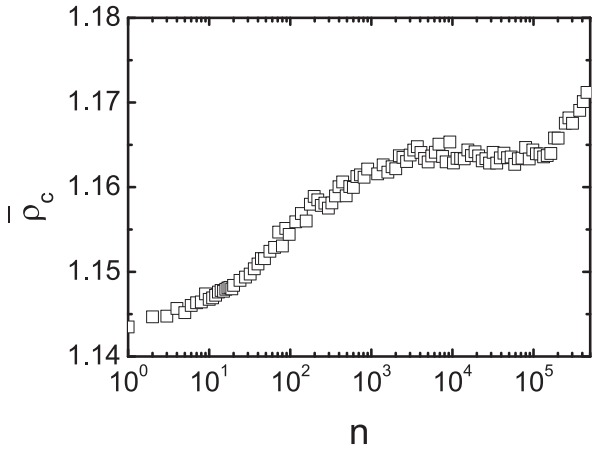

FIG. 10. Relation between average density of nuclei $\bar{\rho}_{c}$ and crystallite size $n$.

the size for $n<1000$. Definitely, at the stage of metastable fluid and crystal nucleation when the nuclei embedded in the system are not yet so big, the increasing crystallite density will be conspicuous with the size. After the nucleus grows to a large enough size, an unexpected phenomenon appears, i.e., its average density almost does not increase any longer (see the plateau appearing in Fig. 10), which is unlike the previous observations of hard spheres. ${ }^{34}$ The possible reason is that the system used in previous simulations is much smaller than ours so as to make the nuclei concerned not that large. Moreover, there is another interesting observation that the crystallite density increases once again when $n>10^{5}$. We infer here that it is probably due to an artifact of finite size effects too. Assuming a nucleus to be spherical, it will feel the image of itself if its diameter exceeds the half box length. So we can estimate the critical number of particles in such a nucleus $N_{c}=\frac{\pi L^{3}}{48} \rho_{c}=\frac{\pi \rho_{c}}{48 N \rho}$, where $L$ is the box length, $\rho \approx 1.14$ (see Fig. 9) is the density of the whole system, and $\rho_{c} \approx 1.163$ (see the plateau value in Fig. 10) is the average density of this nucleus. Then immediately we get to know that a nucleus whose number of crystal particles exceeds $6.7 \times 10^{4}$ will feel the image of itself so as to produce an artifact. For $n>6.7 \times 10^{4}$, the artifact of "self-interaction" will become more and more significant with the growth of nucleus and eventually leads to the self-adjusting so as to make its average density increase again.

In all, the role of density during the whole crystallization process of LJ liquids seems to be distinct, although the variation of density is less significant than that in hard spheres. First, the system gets into a metastable fluid. Some precursors with a relatively high orientational order form and simultaneously, the density of these precursors will increase slightly. Then the crystals emerge inside the precursors but their local density hardly changes. When the crystal nucleation happens, the nucleus size will continue to grow to critical size; meanwhile, the average density of crystal nuclei will increase. During the growth of post-critical nucleus, the average crystal density will increase up to a fixed value and then keep constant. Such a constant density is in fact remarkably smaller than the bulk crystals. Therefore, the crystal nucleation of the LJ system occurs at a density smaller than the bulk crystal density and the subsequent crystal growth cannot make the crystal density increase to a bulk crystal density either. It is possible that a bulk density has to be obtained by other ways in the late period of crystallization such as nucleus coalescence, system rearrangement, etc.

\section{Growth rate}

The crystal growth rate is the growth velocity of the crystalliquid interface. In colloidal experiment, the temporal evolution of the effective radius of the growing post-critical nucleus often approximately shows a linear curve. ${ }^{37}$ By plotting the data of growing crystallites and then doing a linear fit to get the slope, one can measure the crystal growth rate. In contrast, the crystal growth rate of the atomic system is almost impossible to be detected by experiment due to its microscopic scale level. Computer simulation, especially molecular dynamics, is a good method to study the growth rate of the atomic system. In former computation of the growth rate, some special simulation methods had to be presented ${ }^{38}$ because the direct simulation of the growing post-critical nucleus required a large system making the computational cost too expensive. Here, we have used the GPU-accelerated algorithm to perform a direct molecular simulation of crystal growth in a very large system, where the post-critical nucleus can grow to a large enough size.

Let us first focus on the post-critical nuclei that are growing up and embedding in the liquids. Notice here that in a typical simulation there is only one post-critical nucleus growing which actually become the largest nucleus at the growth stage. Then we trace all the trajectories of post-critical nuclei, including their formation and growth, as well as calculate their extending size. Here, we define the growth rate $v=d R_{\text {eff }} / d t$, where $R_{\text {eff }}=\sqrt[3]{3 V_{\text {nucleus }} / 4 \pi}$ is the effective radius of the growing post-critical nucleus. In Fig. 11, we show the temporal evolution of $R_{\text {eff }}$ in a typical MD simulation (corresponding to Fig. 1). Obviously, the emergence of such a nucleus that subsequently grows to a critical size and can continue to grow up happens at $t \approx 2.3 \times 10^{5} \delta t$. When $t \approx 2.4 \times 10^{5} \delta t$, the nucleus seems to reach a critical size, but its growth rate is very small because the rate difference between crystal addition and subtraction is almost zero. After the post-critical nucleus grows to a rather large size which makes the nucleus growth irreversible, the relationship between the size of the post-critical nucleus and time appear to become approximately linear so that the growth rate can be obtained by a linear fit (see the solid line in Fig. 11). After doing the linear fit for all the $74 \mathrm{MD}$ trajectories that succeed in crystallization, we can obtain the averaged growth rate $\bar{v}=0.2$.

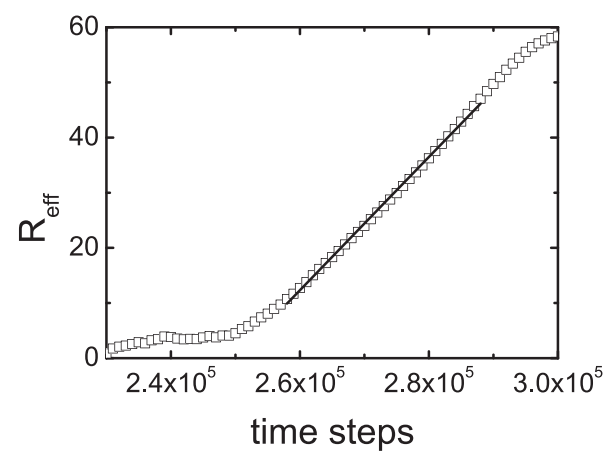

FIG. 11. Temporal evolution of the effective nucleus radius for a typical crystallization process corresponding to Fig. 1. Solid line represents the linear fit to the data of $R_{\text {eff }}$. The slope of the linear fit is $b=1.2 \times 10^{-3}$; so the growth rate is $v=b / \delta t=0.24$. 
Theoretically, the crystal growth rate is expressed as

$$
v(T)=K(T)[1-\exp (-\beta \Delta \mu)],
$$

where $\Delta \mu$ is the chemical potential difference between the liquid and crystal phases. For the crystallization of the LJ system, Burke et al. observed in 1980s that the growth rate was anisotropic. ${ }^{39}$ The growth of the (110) and (100) crystal surfaces is interpreted as a collisioncontrolled scenario, i.e., $K(T)=(a / \lambda)\left(3 k_{B} T / m\right)^{1 / 2} f$, where $\lambda=0.4 a$ and $f=0.27 .{ }^{39}$ On the other hand, the growth rate of the (111) crystal surface that is relevant to the formation of hcp is diffusion-controlled and can be described as the Wilson-Frenkel expression; so $K(T)=6 D f / l$, where $D$ is the diffusion coefficient at the crystal-liquid interface (approximated as diffusion coefficient in liquid, see the bottom of Fig. 1) and $l=\sigma \phi^{-1 / 3}$ is the thickness of a particle layer. ${ }^{37}$ From the above formulas, we can estimate the growth rates of both collision-controlled kinetics and diffusioncontrolled kinetics at $T=1.44$ and $P=21.23$. We have found that the averaged growth rate we obtain from MD simulations is lower than that of collision-controlled kinetics but much larger than that of diffusion-controlled kinetics. As the crystal-liquid interface during actual crystal growth is roughly spherical and the crystals on the interface are the mixture of fcc, hcp, and bcc, it is possible that the crystal growth is the combination of both diffusion-controlled kinetics and collision-controlled kinetics.

\section{CONCLUSION}

We have performed large-scale MD simulations to investigate the entire crystallization process of LJ liquids. By implementing a GPU-accelerated parallel algorithm on a CUDA platform, we are able to use more than $10^{6}$ particles to efficiently study the crystal nucleation and growth. Trying to avoid the coalescence of nuclei in order for an explicit observation of the crystallization process, we set a supercooling that makes only one critical nucleus emerge and grow. As expected, the crystallization of LJ liquids is typically a precursor-mediated process. Although the precursors that could play a role in determining the polymorph are predominantly bcc ordered, the polymorph selection at different stages is rather different. At the stage of metastable fluid, the fraction of bcc is comparable to that of dominant fcc, while the fraction of hcp is the smallest. After the critical nucleus is formed and continue to grow up, bcc particles mostly scatter on the surface of the growing post-critical nucleus, which is in accord with the previous observations ${ }^{18,19}$ and is considered as a manifest of Ostwald's step rule. ${ }^{32}$ With the crystal growth, the fraction of hcp increases to be larger than that of bcc and is eventually comparable to that of fcc.

We study in detail the role of density in the crystallization of LJ liquids. The precursors that have a relatively high orientational order are on average in a denser region than uniform liquids as there is a weakly linear coupling relationship between the average density $\bar{\rho}$ and $Q_{6}$. However, the regions of high density are not necessary on average to have a high orientational order suggesting that the crystal nucleation is not sure to happen in the densest regions, as some confocal experiments have observed in colloids. ${ }^{40,41}$ When the precursors transform into crystals, on average there is almost no density change indicating that the liquid-solid transition microscopically involves a continuous density. More interestingly, the average density of nuclei first seems to increase with the crystal growth and then almost keeps constant after the nucleus reaches a large enough size. After all, the density variation of LJ crystallization is more apparent than that of soft particles but a bit less significant than that of hard spheres. Thus one may be curious to know whether such a similar phenomenon also happens in the crystallization of hard spheres, which would call for another study of large-scale simulation.

With large enough system size, the growth rate is able to be directly calculated by using the linear fit to the temporal evolution of the growing nucleus radius. The value of the growth rate is between that of collision-controlled kinetics and diffusion-controlled kinetics. Due to the anisotropy of crystal growth in the Lennard-Jones system, it can be expected that the polymorph selection, especially the structures of the crystal-liquid interface have an important role in the growth rate. In the actual crystallization process, the region of interface is often the mixture of fcc, hcp, and bcc crystals, so we may assume that a typical crystal growth is driven by both collision-controlled and diffusion-controlled mechanisms.

\section{ACKNOWLEDGMENTS}

Wenze Ouyang would like to thank Dr. Alexander Stukowski for the kind help in some skills of the software Ovito. We are also heartily grateful to Dr. You-Liang Zhu for fruitful discussions in the improvement of the GPU parallel algorithm. This work was supported by the National Natural Science Foundation of China (Grant Nos. 11572322 and 11672295) Programs and the Key Research Program of Frontier Sciences, CAS (Grant No. QYZDY-SSW-JSC040).

\section{REFERENCES}

${ }^{1}$ K. Kelton and A. Greer, Nucleation in Condensed Matter: Applications in Materials and Biology (Elsevier, Amsterdam, 2010).

${ }^{2}$ G. C. Sosso, J. Chen, S. J. Cox, M. Fitzner, P. Pedevilla, A. Zen, and A. Michaelides, Chem. Rev. 116, 7078 (2016).

${ }^{3}$ J. Russo and H. Tanaka, J. Chem. Phys. 145, 211801 (2016).

${ }^{4}$ D. Frenkel and B. Smit, Understanding Molecular Simulation: From Algorithms to Applications, 2nd ed. (Academic Press, San Diego, 2001).

${ }^{5}$ J. D. Honeycutt and H. C. Andersen, J. Phys. Chem. 90, 1585 (1986).

${ }^{6}$ W. C. Swope and H. C. Andersen, Phys. Rev. B 41, 7042 (1990).

${ }^{7}$ H. E. A. Huitema, J. P. van der Eerden, J. J. M. Janssen, and H. Human, Phys. Rev. B 62, 14690 (2000).

${ }^{8}$ F. H. Streitz, J. N. Glosli, and M. V. Patel, Phys. Rev. Lett. 96, 225701 (2006).

${ }^{9}$ J. P. Hansen and I. R. Mcdonald, Theory of Simple Liquids, 3rd ed. (Academic Press, Oxford, 2006).

${ }^{10}$ R. Agrawal and D. A. Kofke, Mol. Phys. 85, 43 (1995).

${ }^{11}$ M. A. van der Hoef, J. Chem. Phys. 113, 8142 (2000).

${ }^{12}$ M. A. Barroso and A. L. Ferreira, J. Chem. Phys. 116, 7145 (2002).

${ }^{13}$ M. P. Allen and D. J. Tildesley, Computer Simulation of Liquids (Clarendon Press, Oxford, 1987).

${ }^{14}$ Y. L. Zhu, H. Liu, Z. W. Li, H. J. Qian, G. Milano, and Z. Y. Lu, J. Comput. Chem. 34, 2197 (2013).

${ }^{15}$ J. Sanders and E. Kandrot, CUDA by Example: An Introduction to GeneralPurpose GPU Programming (Addison-Wesley, San Francisco, 2011).

${ }^{16}$ H. J. C. Berendsen, J. P. M. Postma, W. F. Vangunsteren, A. Dinola, and J. R. Haak, J. Chem. Phys. 81, 3684 (1984).

${ }^{17}$ P. J. Steinhardt, D. R. Nelson, and M. Ronchetti, Phys. Rev. B 28, 784 (1983).

${ }^{18}$ P. R. ten Wolde, M. J. Ruiz-Montero, and D. Frenkel, Phys. Rev. Lett. 75, 2714 (1995).

${ }^{19}$ P. R. ten Wolde, M. J. Ruiz-Montero, and D. Frenkel, J. Chem. Phys. 104, 9932 (1996). 
${ }^{20}$ W. Mickel, S. C. Kapfer, G. E. Schröder-Turk, and K. Mecke, J. Chem. Phys. 138, 044501 (2013)

${ }^{21}$ S. Arai and H. Tanaka, Nat. Phys. 13, 503 (2017).

${ }^{22}$ W. Lechner and C. Dellago, J. Chem. Phys. 129, 114707 (2008).

${ }^{23}$ J. Russo and H. Tanaka, Soft Matter 8, 4206 (2012).

${ }^{24}$ A. Stukowski, Modell. Simul. Mater. Sci. Eng. 18, 015012 (2010).

${ }^{25}$ H. Tanaka, Eur. Phys. J. E 35, 113 (2012).

${ }^{26}$ W. Z. Ouyang, C. L. Fu, Z. W. Sun, and S. H. Xu, Phys. Rev. E 94, 042805 (2016).

${ }^{27}$ W. Z. Ouyang, Z. W. Sun, J. Zhong, H. W. Zhou, and S. H. Xu, Sci. China: Chem. 59, $316(2016)$

${ }^{28}$ X. Q. Ji, Z. W. Sun, W. Z. Ouyang, and S. H. Xu, J. Chem. Phys. 148, 174904 (2018).

${ }^{29}$ C. Desgranges and J. Delhommelle, J. Am. Chem. Soc. 128, 10368 (2006).
${ }^{30}$ C. Desgranges and J. Delhommelle, Phys. Rev. Lett. 98, 235502 (2007).

${ }^{31}$ H. Wang, H. Gould, and W. Klein, Phys. Rev. E 76, 031604 (2007).

${ }^{32}$ W. Ostwald, Z. Phys. Chem. (Muenchen, Ger.) 22, 289 (1897).

${ }^{33}$ J. T. Berryman, M. Anwar, S. Dorosz, and T. Schilling, J. Chem. Phys. 145, 211901 (2016).

${ }^{34} \mathrm{~J}$. Russo and H. Tanaka, Sci. Rep. 2, 505 (2012).

${ }^{35}$ Y. W. Li and Z. Y. Sun, Soft Matter 12, 2009 (2016).

${ }^{36}$ C. P. Royall and S. R. Williams, Phys. Rep. 560, 1 (2015).

${ }^{37}$ Z. R. Wang, F. Wang, Y. Peng, and Y. L. Han, Nat. Commun. 6, 6942 (2015).

${ }^{38}$ J. J. Hoyt, M. Asta, and A. Karma, Interface Sci. 10, 181 (2002).

${ }^{39}$ E. Burke, J. Q. Broughton, and G. H. Gilmer, J. Chem. Phys. 89, 1030 (1988).

${ }^{40}$ P. Tan, N. Xu, and L. Xu, Nat. Phys. 10, 73 (2014).

${ }^{41}$ Y. Z. Lu, X. Lu, Z. X. Qin, and J. Shen, Solid State Commun. 217, 13 (2015) 